\title{
Ethylene Dichloride Production by Oxychlorination in a Fluidized Bed Reactor with CFD Model
}

\author{
Rawinun Junsittiwate ${ }^{1}$, Anoma Kodchakong ${ }^{1}$ and Thongchai Rohitatisha Srinophakun ${ }^{1,2, *}$ \\ ${ }^{1}$ Department of Chemical Engineering, Faculty of Engineering, Kasetsart University \\ Bangkok, Thailand.

\begin{abstract}
${ }^{2}$ Center of Excellence for Petroleum, Petrochemicals, and Advanced Materials; Center for Advanced Studies in Industrial Technology, Department of Chemical Engineering Bangkok, Thailand.

*Corresponding author's email: fengtcs [AT] hotmail.com
\end{abstract}

\begin{abstract}
The flow behavior of 3-dimensional gas-solid fluidized bed reactors is investigated. Computational Fluid Dynamics (CFD) based on the Eulerian-Eulerian flow model is simulated for ethylene dichloride production in an oxychlorination unit as an industrial case study. The simulated riser portion is $3.9 \mathrm{~m}$ in diameter and $15.5 \mathrm{~m}$ in height. The simulation is separated into 2 models; constant catalyst volume fraction and catalyst load variation. The effects of the operating parameters are studied. The ethylene molar feed fraction is varied from 0.17 to 0.3 with the constant mole ratio of hydrochloric acid to oxygen at 3.6. The result shows that the ethylene conversion gradually decreases when the ethylene mole fraction of the feed is more than 0.22. With the outlet pressure rising from 200 to $450 \mathrm{kPa}$, the ethylene conversion increases from 96.39 to $98.46 \%$. The ethylene conversion significantly increases in the pressure range from 200 to $400 \mathrm{kPa}$ and stays constant at over $400 \mathrm{kPa}$. The increase of cooling medium temperature from 320 to $420 \mathrm{~K}$ drives the ethylene conversion from 96.9 to $98.8 \%$ and the gas outlet temperature from 422 to $565 \mathrm{~K}$. The gas feed temperature is varied from 400 to $500 \mathrm{~K}$. The simulation shows that the gas feed temperature is varied from 400 to $500 \mathrm{~K}$. The simulation shows that the gas outlet temperature also increases from 476 to $527 \mathrm{~K}$ but the ethylene conversion has a minor effect. Variation of catalyst quantity from 19,500 to 78,000 $\mathrm{kg}$ is carried out to study gas-solid flow behavior and transport phenomena. The simulation of 78,000 $\mathrm{kg}$ of catalyst shows the blowing out effect. The developed CFD model has the potential of being applied as a tool to predict the effect of the operating parameters and the gas-solid behavior in the reactor.
\end{abstract}

Keywords - Computational Fluid Dynamics, Oxychlorination

\section{INTRODUCTION}

In recent years, a number of studies have been carried out in order to improve the understanding of various phenomena taking place in fluidized-bed reactors. Many of them have generated models whose application has very often been restricted to specific conditions, e.g. isothermal processes or simple linear kinetics or fixed bubble diameter. Despite these continuous efforts, there has not been much success in setting up a highly reliable model for the design of catalytic fluidized-bed reactors. Therefore, the task of designing or scaling up a fluidized-bed reactor must still proceed through costly and time-consuming empirical methods [1]. Relying on such empirical procedures in scale-up, one should always be prepared for unexpected problems emerging from the lack of sufficient understanding of the nature of fluidized beds.

Computational fluid dynamic (CFD) has become a favored engineering tool to predict flow in various field because it is convenient and reduce time consumption. Using the CFD method, including both computational and modeling issues, it has been demonstrated that detailed mechanically based numerical simulations of two-phase flows and heat transfer are feasible with existing CFD codes such as CFX 5.6. The computational model describes both the continuous phase and the dispersed phase using a so-called Eulerian model. In these models the dispersed phase, like the continuous phase, is described as a continuous fluid with appropriate closures. Hence, one calculates only the average local volume fraction, velocity, etc. and not the properties of each dispersed fluid or droplet [2].

The purpose of this paper is to present the hydrodynamics three-dimensional gas-solid flow in ethylene oxychlorination in a fluidized bed reactor. Ethylene dichloride (EDC) is used to manufacture vinyl chloride monomer (VCM), which is one of the largest chemical commodities produced in the world. EDC may be produced by the direct 
chlorination of ethylene or oxychlorination of ethylene in the presence of oxygen and hydrogen chloride. Around $85 \%$ of the total EDC production is used for the production of vinyl chloride. $10 \%$ is used in the production of chlorinated solvents such as 1,1,1-trichloroethane and tri- and tetrachloroethylene. The rest goes into various processes, mainly for synthesis of ethylenediamines [3]. The purity of EDC used for cracking to VCM is about 96-99\%wt since the cracking process is extremely sensitive to inhibition and fouling by trace amounts of impurities or additives. Some of them can act either as promoters or as inhibitors, which cause undesirable coke formation.

Oxychlorination is a catalytic reaction of ethylene with hydrogen chloride and oxygen to form EDC. In the oxychlorination reactor, ethylene reacts with dry $\mathrm{HCl}$ and either air or pure oxygen in a heterogeneous catalytic reaction (Oxychloro VIII as a catalyst) at operating conditions of about $227^{\circ} \mathrm{C}$ and $300 \mathrm{kPa}$ to form ethylene dichloride $\left(\mathrm{C}_{2} \mathrm{H}_{4} \mathrm{C}_{12}\right)$ and water. The overall chemical reaction is

$$
\begin{gathered}
\mathrm{C}_{2} \mathrm{H}_{4}+2 \mathrm{HCl}+0.5 \mathrm{O}_{2} \rightarrow \mathrm{C}_{2} \mathrm{H}_{4} \mathrm{Cl}_{2}+\mathrm{H}_{2} \mathrm{O} \\
-\Delta \mathrm{H}_{\mathrm{r}}^{\mathrm{o}}=295 \mathrm{~kJ} / \mathrm{mol}
\end{gathered}
$$

The kinetic rate in the reactor for this work for the process of ethylene dichloride is reported by [4] formation and the rate has been used in the work of [5]. The kinetic condition of the first-order reaction, which depends on the concentration of cupric chloride related to the concentration of ethylene, is interpreted by a Langmuir-Hinshelwood mechanism; however, the results of the reaction may occur by oxychlorination of ethylene. Cupric chloride reacts with $\mathrm{HCl}$ and $\mathrm{O}_{2}$ to produce the copper salt. The reaction rate is shown as follows:

$$
\mathrm{r}=\frac{\mathrm{k}_{\mathrm{r}} \mathrm{K}_{\mathrm{a}} \mathrm{C}_{\mathrm{E}} \mathrm{C}_{\mathrm{c}}}{1+\mathrm{K}_{\mathrm{a}} \mathrm{C}_{\mathrm{E}}}
$$

Where $\mathrm{k}_{\mathrm{r}}=269 \exp (-37.8 / \mathrm{RT}), \mathrm{K}_{\mathrm{a}}=0.63$

The Construction of a large-scale industrial OXY reactor is extremely complex. The reactor has a wide range of length scales, starting from a few meters (reactor diameter and length) to a few millimeters (orifice of mixing element). Fluid flow in the reactor produces a lot of heat from exothermic reaction so the cooling appears to be important. There are two/three orders of magnitude difference between gas velocities in the various elements of an OXY reactor. Resolving these widely different length and velocity scales (typically in the range 0.01 to $4 \mathrm{~m}$ and 0.2 to $100 \mathrm{~m} / \mathrm{s}$ ) simultaneously may stretch the limits of computational resources [6]. Therefore, this work considers only the cooling region of the OXY reactor to evaluate possible misdistributions. The bottom conical portion, grid containing several mixing elements, feed distributors, several internal supports, etc. are not considered and assumed to be of uniform inlet velocity.

The present work describes the results of numerical modeling of a gas-solid fluidized bed with an oxychlorination reaction. The mathematical model is based on Eulerian-Eulerian multiphase flow which applies for the two-phase gassolid flow. That means the laws of conservation of mass and momentum are satisfied by each phase individually. Standard governing equations for single-phase flow (mass and momentum conservation equations) [7] are used as shown in Table 1. Considering the magnitude of pressure drop across the grid, it is necessary to employ the compressible flow equations. An ideal gas assumption is used to calculate density of gas at any point (as a function of local pressure, temperature and effective molecular weight of gas). Under the usual operating conditions, the flow is found to be turbulent. Turbulent stresses are modeled using the $\mathrm{k}-\varepsilon$ model. The following dependent variables are solved for each phase separately. 
Table 1: Governing conservation equations

\section{Continuity Equation}

(for both phase)

\section{Momentum Equation}

(for gas phase)

\section{Momentum Equation}

(for solid particle)

\section{Energy Equation}

$$
\frac{\partial}{\partial t}\left(r_{\alpha} \rho_{\alpha}\right)+\nabla \bullet\left(r_{\alpha} \rho_{\alpha} U_{\alpha}\right)=S_{M S \alpha}+\sum_{\beta=1}^{N_{p}} \Gamma_{\alpha \beta}
$$

$$
\begin{aligned}
\frac{\partial}{\partial t}\left(r_{\alpha} \rho_{\alpha} U_{\alpha}\right)+\nabla \bullet\left(r_{\alpha}\left(\rho_{\alpha} U_{\alpha} \otimes U_{\alpha}\right)\right) \\
=-r_{\alpha} \nabla \rho_{\alpha}+\nabla \bullet\left(r_{\alpha} \mu_{\alpha}\left(\nabla U_{\alpha}+\left(\nabla U_{\alpha}\right)^{T}\right)\right) \\
+\sum\left(\Gamma_{\alpha \beta}^{+} \mathrm{U}_{\beta}-\Gamma_{\beta \alpha}^{+} \mathrm{U}_{\alpha}\right)+\mathrm{S}_{\mathrm{M \alpha}}+\mathrm{M}_{\alpha} \\
\frac{\partial}{\partial t}\left(r_{\alpha} \rho_{\alpha} U_{\alpha}\right)+\nabla \bullet\left(r_{\alpha}\left(\rho_{\alpha} U_{\alpha} \otimes U_{\alpha}\right)\right)
\end{aligned}
$$

$$
=\sum_{\beta=1}^{\mathrm{N}_{\mathrm{p}}}\left(\Gamma_{\alpha \beta}^{+} \mathrm{h}_{\beta \mathrm{S}}-\Gamma_{\beta \alpha}^{+} \mathrm{h}_{\alpha \mathrm{S}}\right)+\mathrm{Q}_{\alpha}+\mathrm{S}_{\alpha}
$$

This paper will present an analysis of the dynamics of fluidized beds and illustrate the application of commercial software, namely CFX, in order to investigate, numerically, the real dimensionality of the reaction in the fluidized bed reactor. The results are compared with data from Thai Plastic and Chemicals plant (TPC) to assess the validity of this model.

\section{SIMULATION}

The differential equations in the mathematical model are solved on a unit volume basis. Thus, the conservations need to yield overall possible finite volumes covering the whole problem space. This method is called finite volume technique. The differential equations express the conservation over infinitesimal control volumes. The 3D geometry with meshing on the symmetrical assumption is generated using ICEM CFD4.CFX software. The computational model is mapped on commercial computational fluid dynamics (CFD) code CFX 5.6 from AEA Technology. The simulation is done on personal computer (AMD Athlon (TM) XP 2400+, $2.00 \mathrm{GHz}$, and 1.50 Gbytes of RAM) with a time stepping of 1 second over a time range up to 200 seconds real time. The detailed simulation parameters from TPC Plant are summarized in Table 2 .

Table 2: Design and operating data used in the simulation

\begin{tabular}{c|c}
\hline Design and Operating data & Value \\
\hline Feed of $\mathrm{C}_{2} \mathrm{H}_{4}$ & $7008 \mathrm{~m}^{3} / \mathrm{hr}$. \\
Feed of $\mathrm{HCl}$ & $13,600 \mathrm{~m}^{3} / \mathrm{hr}$. \\
Feed of $\mathrm{O}_{2}$ & $3,751 \mathrm{~m}^{3} / \mathrm{hr}$. \\
Catalyst in Reactor & $78,000 \mathrm{~kg}$ \\
Catalyst diameter & $51 \mu \mathrm{m}$. \\
Catalyst density & $1,369 \mathrm{~kg} / \mathrm{m}^{3}$ \\
Inlet Pressure & $3.66 \mathrm{~kg} / \mathrm{cm}^{2} \mathrm{G}$ \\
Outlet Pressure & $3.00 \mathrm{~kg} / \mathrm{cm}^{2} \mathrm{G}$ \\
Feed temperature & $177^{\circ} \mathrm{C}$ \\
Operating temperature & $220-227^{\circ} \mathrm{C}$ \\
\hline
\end{tabular}

The geometry of the riser section used in the present simulation is shown in Figure 1. The interesting zone for simulation is the portion where the gas feed from the distributor until the gas passes out of the cooling zone. The size of the simulated portion is $15.5 \mathrm{~m}$. in height and $3.9 \mathrm{~m}$. in diameter (the overall reactor is $31.6 \mathrm{~m}$ in height in from the ground to the top and $3.9 \mathrm{~m}$ in diameter) and the inner cylinder consists of 284 cooling tubes which are $12.2 \mathrm{~m}$. high cylindrical columns with a $9 \mathrm{~cm}$. diameter. The cooling tubes are installed $1.5 \mathrm{~m}$. above a distributor. The 3D geometry of the reactor with meshing on the symmetrical assumption is generated [8]. 


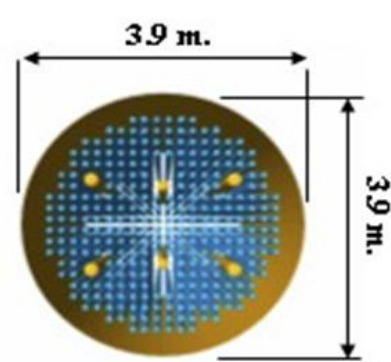

PLAN

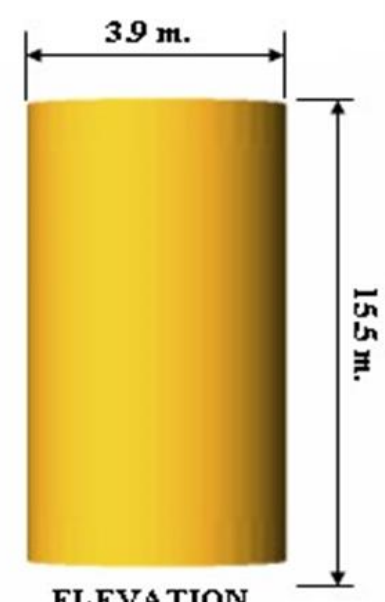

ELEVATION

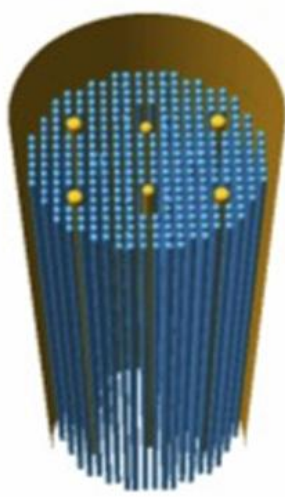

PERSPECTIVE

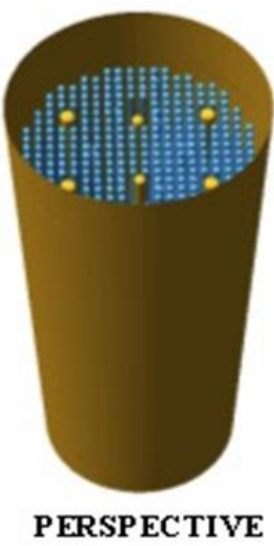

Figure 1: Schematic drawing of 3D reactor

Figure 2 illustrates the grid system with 2 kinds of mesh; the tetrahedral mesh and the prismatic mesh. The grids for a quarter of the reactor consist of approximately 500,000 elements (normal size $9 \mathrm{~cm}$ and maximum size $11 \mathrm{~cm}$ ). Prism meshing gives layers of flat prismatic (Wedge-shaped) elements which provide a smaller mesh length scale in the direction perpendicular to the wall. This provides better resolution of the velocity field near the wall, which is changing rapidly.

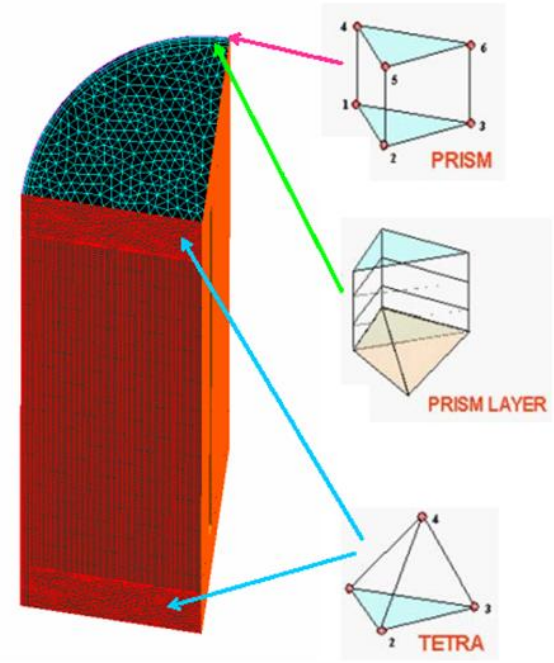

Figure 2: Grid system and schematic drawing of riser portion

The simulation is carried out in two categories; the constant catalyst volume fraction and the variation of catalyst load, to study the gas-solid behavior. The constant of the volume fraction case is studied instead of studying the real catalyst movement of $78,000 \mathrm{~kg}$ to avoid the blowing out effect. Basically, the simulation of 78,000 kg of catalyst needs 
the circulating portion to recycle the catalyst to the reactor to maintain the catalyst quantity in the reactor. This simulation considers only the riser portion; therefore, the assumption of constant catalyst volume fraction is suitable. The variation of catalyst from 19,500 to 78,000 kg is studied for the second category. These models are based on the same assumption. However, the first one includes constant catalyst volume fraction assumption while any other remains the same as summarized below:

- Symmetry in a quarter of the reactor

- Reaction in oxychlorination unit occurs only in simulated portion

- Reactor and cyclone walls are adiabatic

- No-slip condition for all rigid wall

- Constant temperature of cooling coil

- Mass transfer between the particles and the gas phase is negligible

\section{CONSTANT VOLUME FRACTION OF CATALYST}

The reactor in this simulation contains $78,000 \mathrm{~kg}$ of catalyst. The catalyst is assumed to have a constant volume fraction of 0.3 for the reactor domain. The pressure is set equal to the real plant, $294 \mathrm{kPa}$ at the outlet and $358 \mathrm{kPa}$ at the inlet, by adding a resistance source term to the momentum equation for the cooling zone. The product temperature is controlled at $500 \mathrm{~K}$ by adjusting the cooling coil to $350 \mathrm{~K}$.

\subsection{Model Validation}

The overall flow model is simulated at normal operating condition of TPC Plant. The gas-solid fluidization simulation is evaluated by comparing the results with plant data shown in Table 3. The simulation result is clearly in good agreement with the plant data.

Table 3: Comparison between model predictions and Industrial data

\begin{tabular}{c|c|c|c}
\hline Condition & Plant data & Simulation & \% Error \\
\hline Inlet Temperature & $450 \mathrm{~K}$ & $450 \mathrm{~K}$ & $0 \%$ \\
Outlet Temperature & $500 \mathrm{~K}$ & $498.54 \mathrm{~K}$ & $0.3 \%$ \\
Inlet Pressure & $3.66 \mathrm{~kg} / \mathrm{cm} 2 \mathrm{G}$ & $3.7 \mathrm{~kg} / \mathrm{cm} 2 \mathrm{G}$ & $1.1 \%$ \\
Outlet Pressure & $3.0 \mathrm{~kg} / \mathrm{cm} 2 \mathrm{G}$ & $3.0 \mathrm{~kg} / \mathrm{cm} 2 \mathrm{G}$ & $0 \%$ \\
Ethylene conversion & $97.27 \%$ & $98.46 \%$ & $1.2 \%$ \\
HCl conversion & $99.62 \%$ & $100.00 \%$ & $0.4 \%$ \\
\hline
\end{tabular}

In this simulation, the gas phase is modeled as a compressible fluid therefore the total pressure in the reactor depends on temperature, density, and mole of substances. The total pressure and temperature distributions on the reactor can be illustrated as Figure 3. Both pressure and temperature gradually decrease along the distance from the inlet. The drop in pressure is because of the effect of resistance of flow resistance. The cooling coils act as resistance for the gas-solid flow probably due to the reduction in pressure of the reactor from 358 to $294 \mathrm{kPa}$. Between 0.1-0.9 of the reactor heights (1.5$14 \mathrm{~m}$. above the distributor), the pressure significantly decreases with the reactor height because the cooling coils in the zone appear to resist the fluid flow. Consequentially, the pressure drops because of resistance from the cooling coils. Finally, the pressure is quite constant in the range of 0.9-1.0 of the reactor heights (14-15.46 m above the distributor) because of no flow inhibitor in this zone. The highest temperature occurs at $554 \mathrm{~K}$ because this reaction is exothermic and this area has the highest concentration of reactants. In the zone between 0.1-0.3 of the reactor heights, the temperature is rapidly decreased by the cooling coils. The temperature is quite constant between $0.3-0.6$ of the reactor height zones because the released energy from the reaction is completely removed by the cooling coils. The reaction rate has little effect at above 0.6 of the reactor heights (more than $9 \mathrm{~m}$ ), therefore the temperature is gradually decreased by the cooling coil and reaches $500.4 \mathrm{~K}$ at the outlet. 


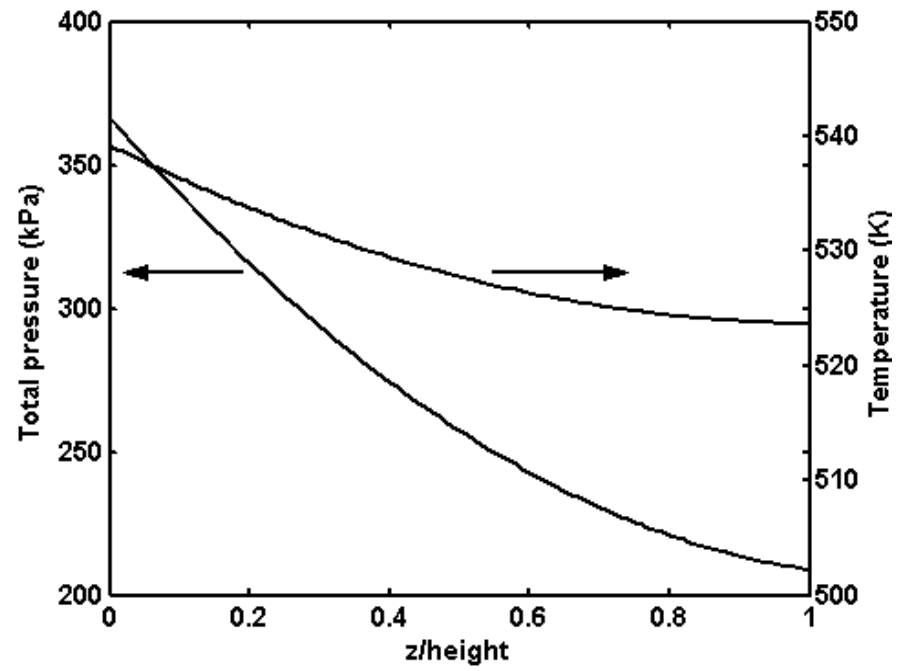

Figure 3: Total pressure and temperature along the reactor height $(0-15.46 \mathrm{~m})$

Figure 4 shows the ethylene conversion as a function of the reactor height. The ethylene and hydrochloric acid conversions decrease along the reactor height. At 0.8 of the reactor heights, the conversion of both ethylene and hydrochloric acid reaches maximum values at $98.46 \%$ and $100 \%$, respectively and stays constant along the distance. The maximum ethylene conversion is limited by the amount of hydrochloric acid.

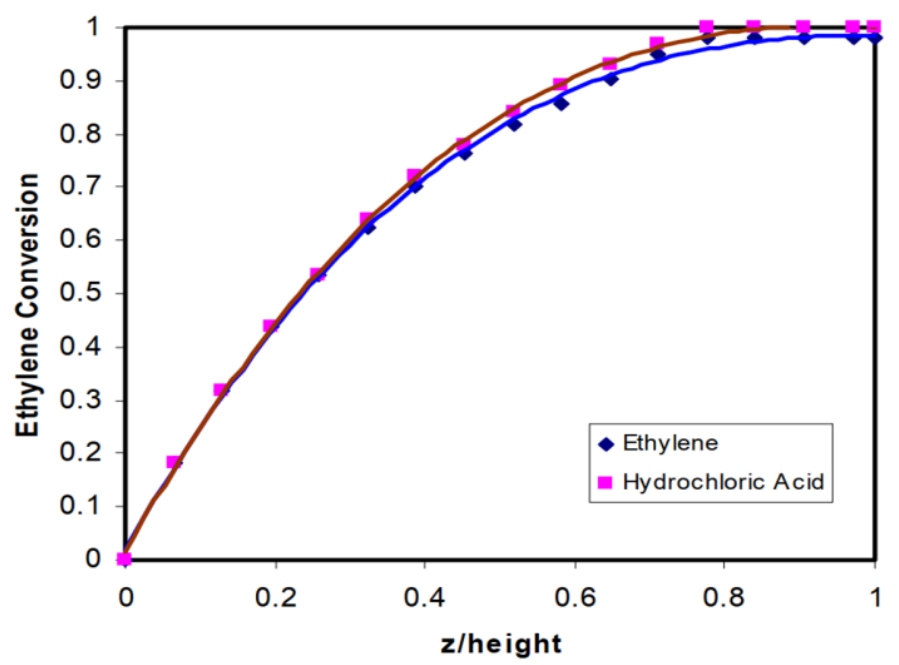

Figure 4: Conversion of ethylene and hydrochloric acid along the reactor height

\subsection{Effect of molar feed distribution}

Molar feed for ethylene is varied from 150 to $300 \mathrm{~kg} \mathrm{~mol} / \mathrm{hr}$. while hydrochloric acid and oxygen feed rate are kept at 555.76 and $153.28 \mathrm{~kg} \mathrm{~mol} / \mathrm{hr}$., respectively. The molar ratio of hydrochloric acid to oxygen is 3.6. Figure 5 shows the relationship between ethylene mole fraction fed and the conversion of ethylene (also the ethylene dichloride production rate), hydrochloric acid, and oxygen. Reaching the highest conversion, the ethylene mole fraction is less than 0.22 . For ethylene conversion above 0.27 , the conversion of hydrochloric acid and the ethylene dichloride production rate remains constant while the ethylene conversion decreases because ethylene is no longer the limiting step. 


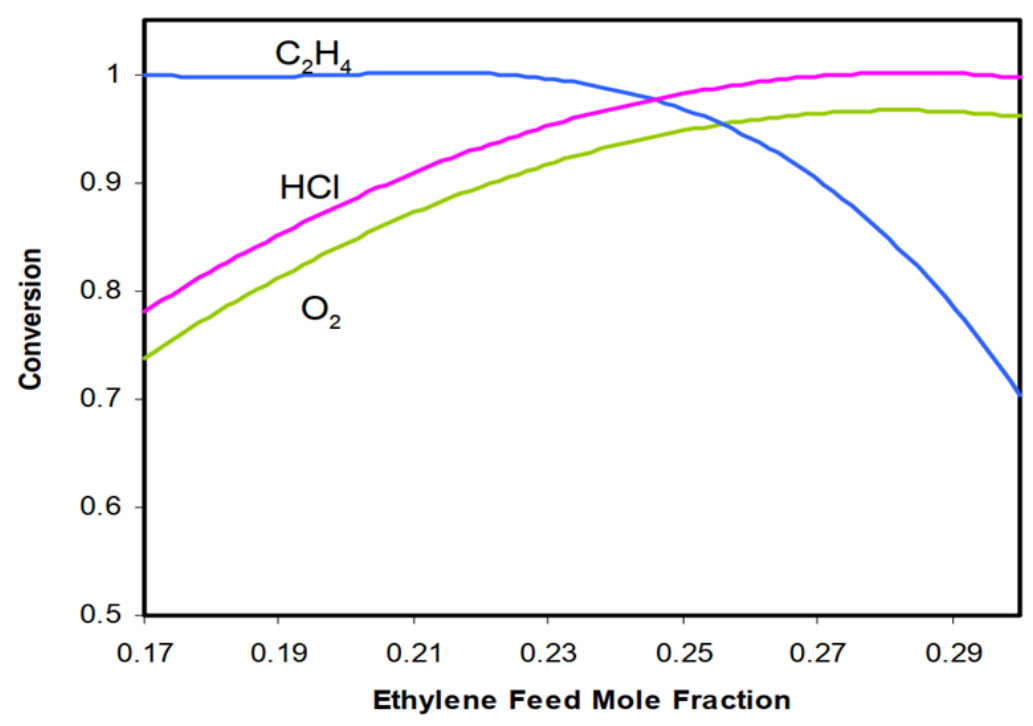

Figure 5: Effect of ethylene mole fraction on conversion

\subsection{Effect of outlet pressure}

The outlet boundary condition is specified in this simulation when a molar feed is constant. The effect of the outlet pressure increment directly causes the increasing of the operating pressure. The results from the simulation show the outlet pressure increase from 200 to $450 \mathrm{kPa}$ leads to a raise in ethylene conversion from 96.39 to $98.46 \%$. Further increase of the outlet pressure above $400 \mathrm{kPa}$ has a slightly effects on the ethylene conversion and consequently ethylene dichloride yield. Pressure is a major influence on the density of gas. The gas density is important because it affects the minimum fluidization and also the bed void age at minimum fluidization. Since the pressure affects the rate of reaction, the pressure is a parameter of ethylene concentration. Thus, increasing pressure obtains high ethylene concentration, therefore the rate of reaction increases.

\subsection{Effect of cooling medium temperature}

The operating temperature of the reactor is controlled by the feed rate of the cooling water; therefore, the effect of the cooling coil temperature is studied. This work simplifies the cooling coil to constant medium temperature at $360 \mathrm{~K}$ to achieve an outlet temperature of $500 \mathrm{~K}$. The effects of cooling medium temperature on the ethylene conversion and outlet temperature are shown in Figure 6. The variation of cooling medium temperature from 300 to $420 \mathrm{~K}$ causes the ethylene conversion to increase from 96.39 to $98.46 \%$. The outlet temperature increases from 422 to $565 \mathrm{~K}$ with the increase of cooling temperature from 300 to $420 \mathrm{~K}$. The cooling medium temperature is a variable parameter of the heat balance equation and any increase in the cooling medium temperature will affect the heat removal term and the value of the product temperature. 


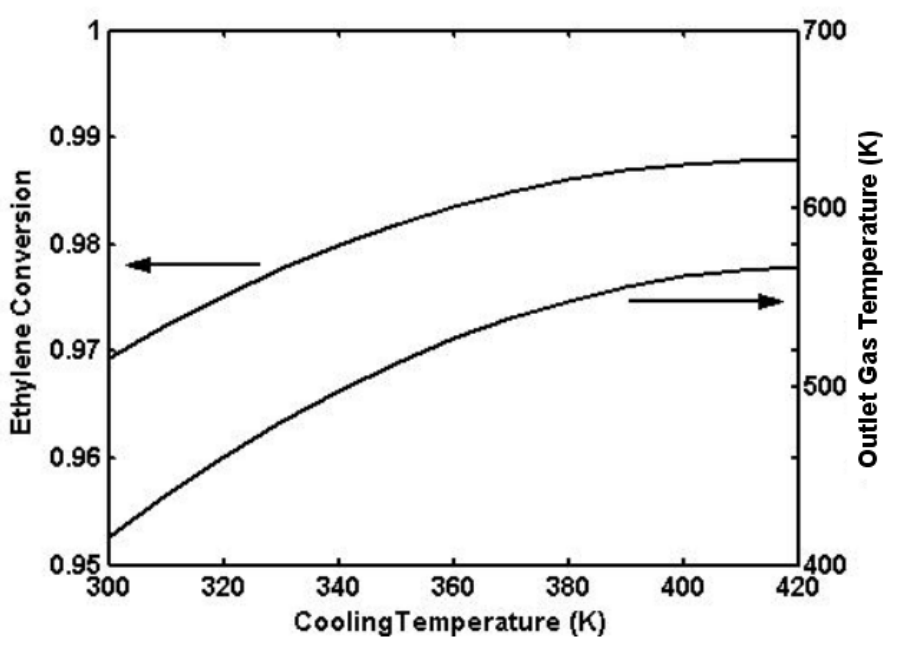

Figure 6: Effect of cooling medium temperature on outlet gas temperature and ethylene conversion

\subsection{Effect of gas feed temperature}

Figure 7 shows the effect of variation of feed temperature on ethylene conversion and product temperature. Feed Temperature is varied from 400 to $500 \mathrm{~K}$. The feed temperature has little effect on conversion. In contrast, it has a major effect on product temperature. It causes the outlet temperature to increase from 476 to $527.067 \mathrm{~K}$. The feed temperature also impacts the diffusivities of components in the gas mixture as shown. The diffusivity of each component affects the mass transfer coefficient. Each mass transfer coefficient increases as the diffusivity increases.

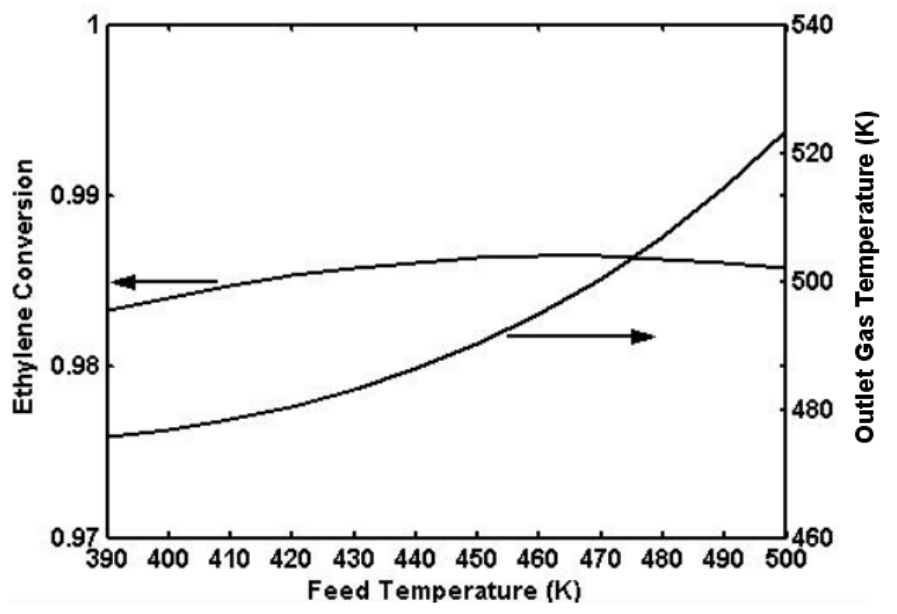

Figure 7: Effect of feed temperature on ethylene conversion

\section{CATALYST LOAD VARIATION}

The efficiency of this mathematical model for the hydrodynamic prediction on the gas-solid flow is shown in this section. The study of catalyst load effect is simulated in 4 cases for 100 seconds each as shown in Table 4 .

Table 4: The catalyst quantity in the reactor

\begin{tabular}{c|c}
\hline Case & Catalyst (kg) \\
\hline 1 & 19,500 \\
2 & 39,000 \\
3 & 58,500 \\
4 & 78,000 \\
\hline
\end{tabular}


Actually, the simulation can describe the hydrodynamic behavior of gas-solid flows as well as the reactions. For instance, Figures 8 to 14 demonstrate the simulation results of case 4 (78,000 kg of catalyst).

Figure 8 captures the catalyst distribution on the middle plane of the reactor at different times. The volume fraction at initial condition is set equal to 0.3 for the portion above the inlet to $80 \%$ of the cooling height. The predicted profile at $20 \mathrm{~s}$ shows the existence of both dense annulus and dilute core which can be easily recognized. The volume fraction in the reactor reduced with time because the catalyst was flowing out.

In case 4 (78,000 kg of catalyst), the gas-solid hydrodynamics are studied from the middle plane at 50 seconds. Figure 9 shows a high density of a dense catalyst at the core of the reactor. The high catalyst populations are also found at the wall region because this zone received less effect from the cooling coil resistance. Figure 9(b) illustrates the distribution of gas temperature. In the bottom zone where reactants are fed, the temperature is higher than other zones. One can notice that surrounding the cooling coils, the temperature is drops. The catalyst velocity profiles are shown in Figure 9(c). The maximum velocity is found at the entrance of the reactor. Swirling and high velocity also appears in the regions before and after the cooling bundle [9].

The pressure is high at the bottom and gradually decreases along the height of the reactor. There is a slight difference of pressure in the radius direction.

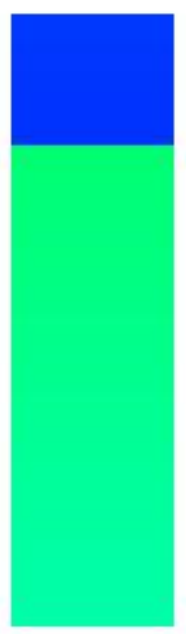

$\mathbf{T}=\mathbf{0 s}$

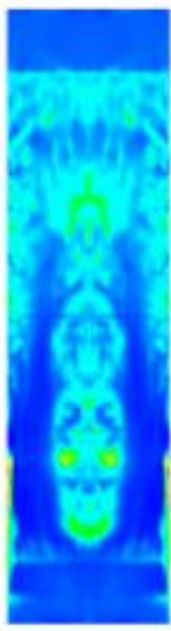

$\mathbf{T}=\mathbf{3 0} \mathrm{s}$

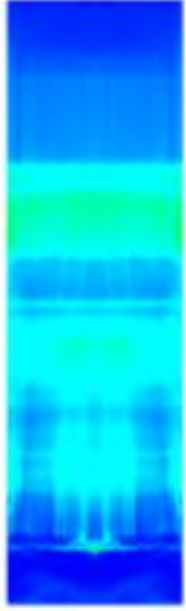

$T=10 \mathrm{~s}$

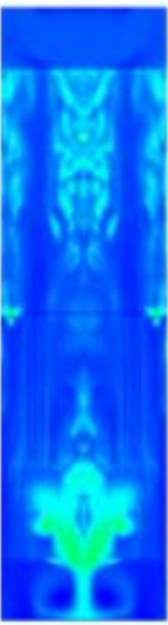

$\mathbf{T}=\mathbf{5 0} \mathrm{s}$

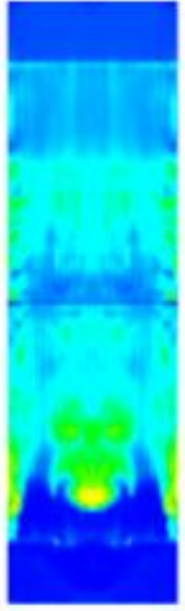

$\mathbf{T}=\mathbf{2 0 ~ s}$

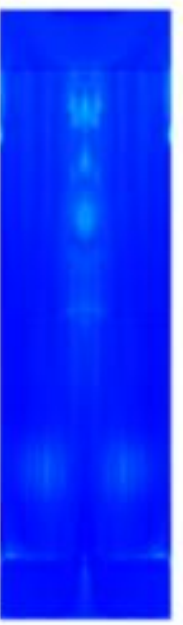

$T=100 \mathrm{~s}$
Catalyst

Volume Fraction

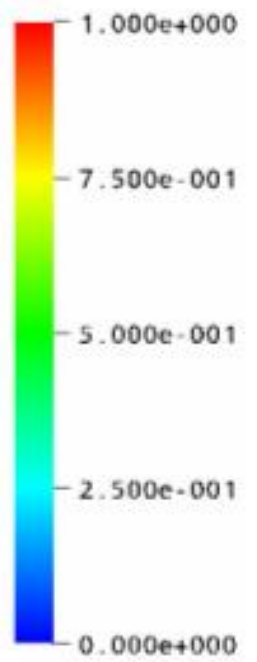

Figure 8: Catalyst distribution for case 4 at the middle plane 

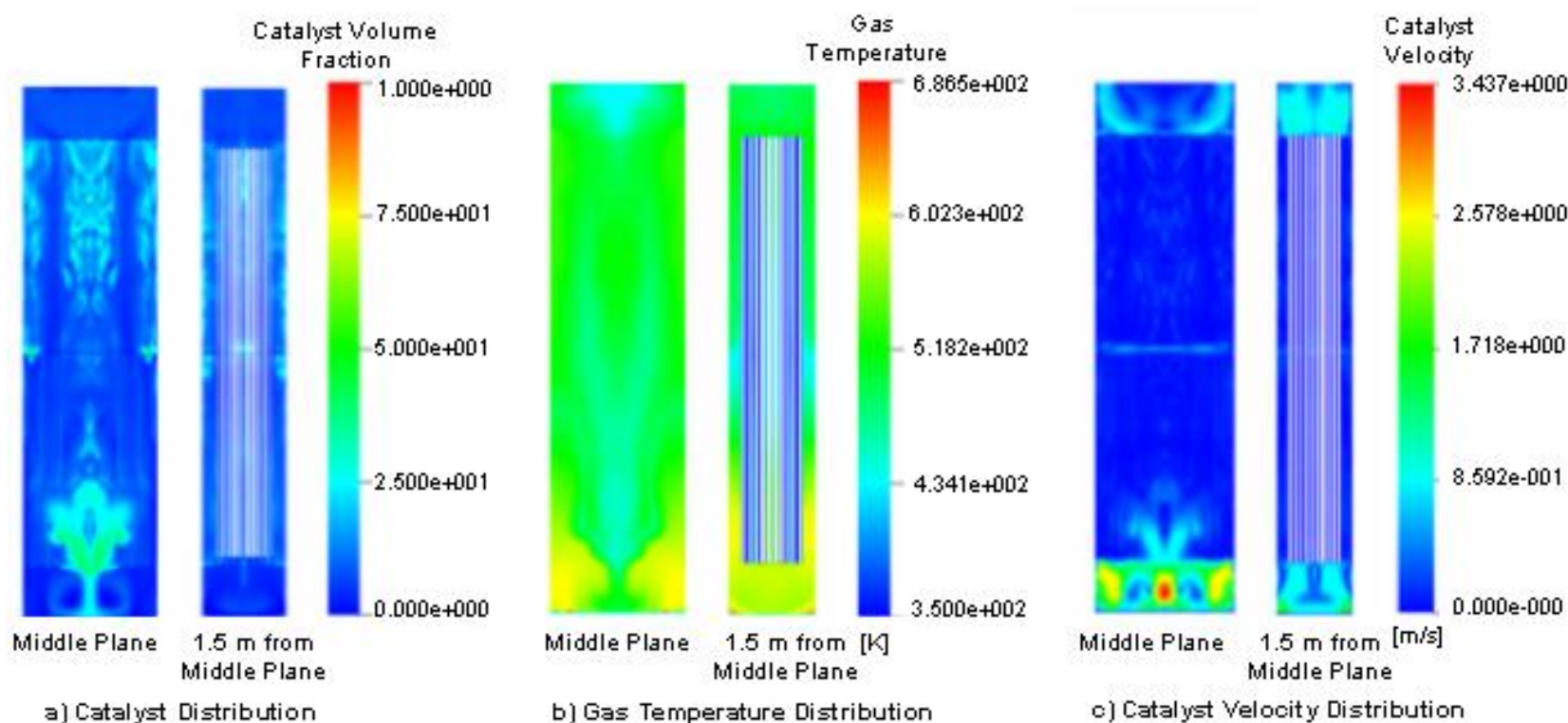

Figure 9: Gas-solid distribution from the middle plane for case 4 at 50 seconds

The cross-section distribution of catalyst at a height of $11 \mathrm{~m}$ is captured from 0-100 seconds as shown in Figure 10. The total amount of catalyst is $78,000 \mathrm{~kg}$ by specify the catalyst volume fraction at 0.3 from the inlet to $11 \mathrm{~m}$ at the initial condition. Referring to the model, the volume fraction increases to 0.5 after 10 seconds due to the force pushing the catalyst up. Then, after 10 seconds, some catalyst is blown off.

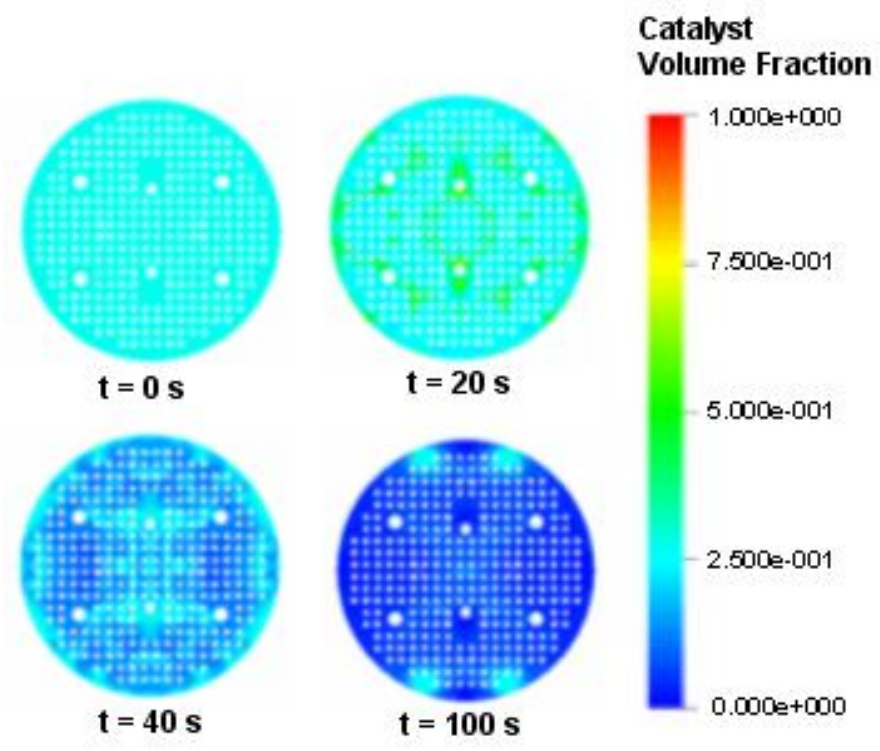

Figure 10: Catalyst distribution on the vertical plane (11 $\mathrm{m}$ above the inlet) for case 4

Figure 11 also shows the temperature distribution in the same section with the initial temperature at $400 \mathrm{~K}$. In the early stage of simulation, the temperature is high because of the exothermic reaction and the gas temperature itself. Therefore, the distribution is high at the start up. After the reaction develops to a steady state, the temperature decreases until it becomes stable. The area close to the cooling coil has a lower temperature due to heat release. In this area, the gas seems to pass a bit lower because of the surface resistance of the coil 


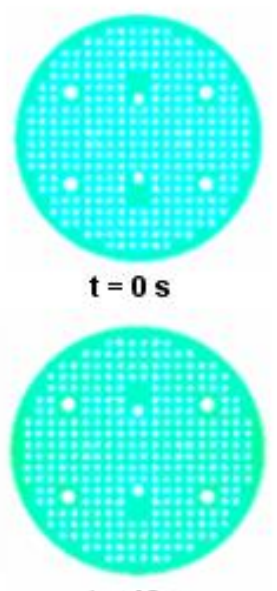

$t=40 s$

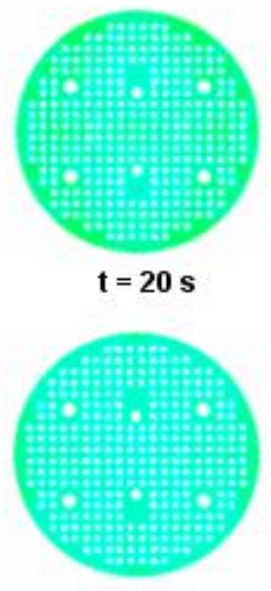

$t=100 s$

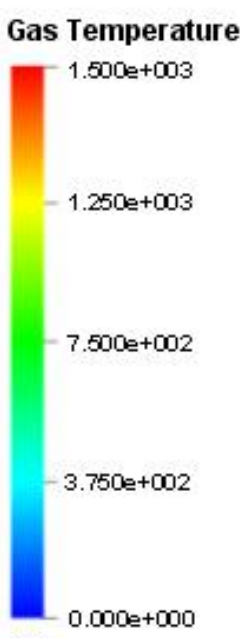

(K)

Figure 11: Temperature distribution on the vertical plane (10 $\mathrm{m}$ above the inlet) for case 4

Figure 12 presents a pressure drop as a function of riser height at 100 seconds. This figure indicates that the pressure drops along the reactor height due to the effect of the flow resistance. However, the comparison of the pressure in each case is not successful because the catalyst, the major factor for pressure drop, is blowing out.

Figure 13 shows the averaged catalyst velocity as a function of the reactor height at 100 seconds. The catalyst volume fraction is significant at the lower zone of the reactor. This is the effect of the gravitational force. The comparison of catalyst volume fraction at 100 seconds found that maximum volume fractions are found at 0.15 of reactor height in case $2(39,500)$. The volume fraction is more than zero for all cases at the outlet $(\mathrm{z} /$ height $=1)$ due to the catalyst blow out effect.

Figure 14 shows the catalyst velocity at 100 seconds. The results are significantly higher in the region below 0.2 and above 0.8 of the reactor heights because this zone has a high resistance as a result of adding the resistance term to compensate for the resistance from supports and assumption of a no slip condition at the cooling wall.

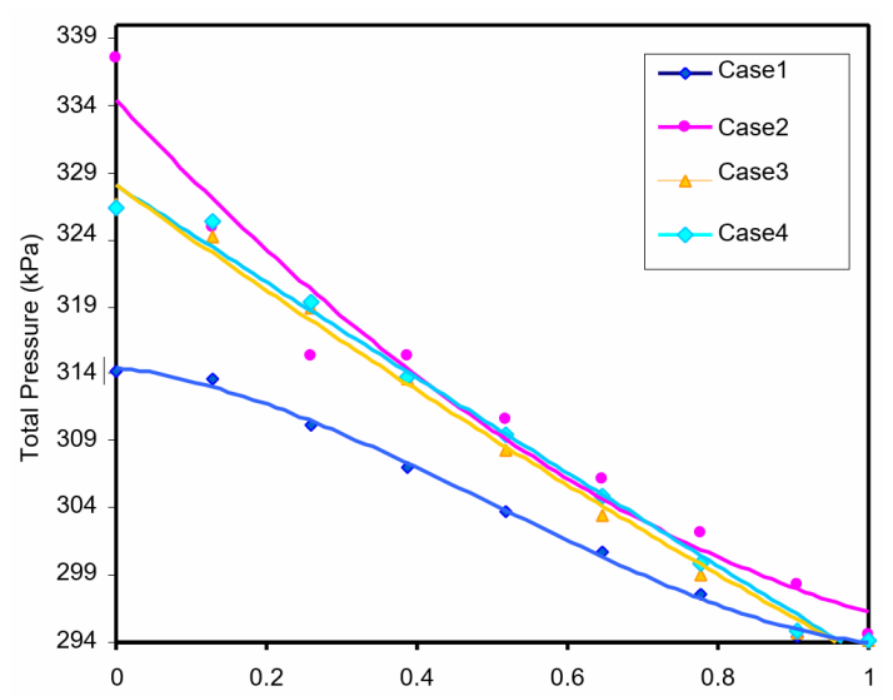

Figure 12: Pressure drop as a function of reactor height at 100 seconds 


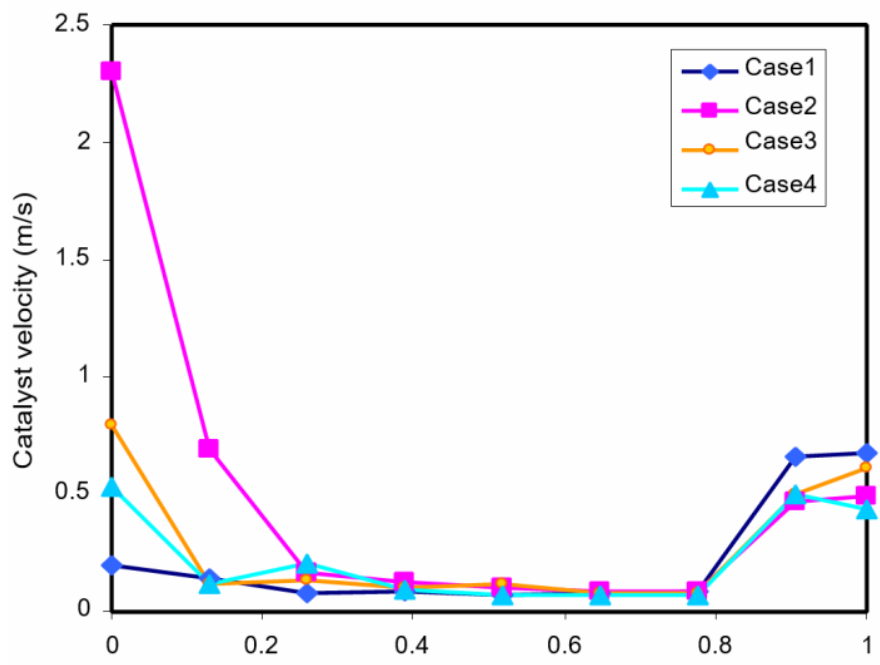

Figure 13: Catalyst volume fraction as a function of reactor height at 100 seconds

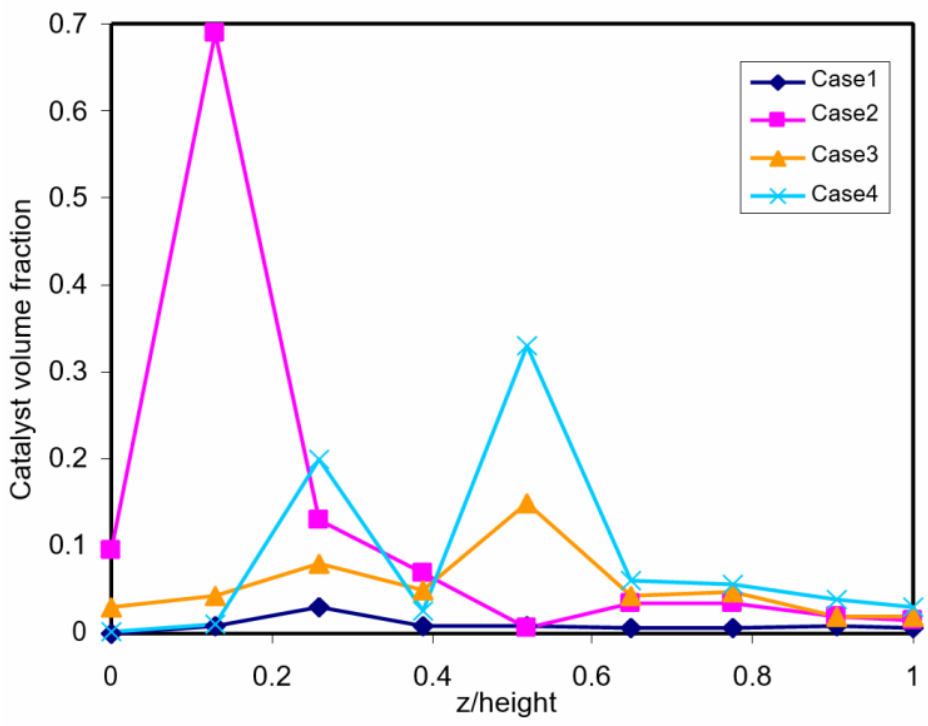

Figure 14: Catalyst velocity as a function of reactor height at 100 seconds

First, the vertical column and each type of inlets are drawn in Design Modeler program. The second step is Mesh, which divides the system into several small volume elements and the next step is Setup. In this process, governing equations, properties of the material and boundary conditions will be defined completely in order to get the simulation results accurately as much as possible. After that, the model is calculated in a solution step. The last step is Results that analyze simulation results by using the CFD-post process.

Moreover, experiments are conducted in a laboratory using a vertical column and various inlets the same as the actual work. Experimental results are recorded in order to check the accuracy of the simulation results.

\section{CONCLUSION}

A mathematical model of the oxychlorination unit for ethylene dichloride $\left(\mathrm{EDC}, \mathrm{C}_{2} \mathrm{H}_{4} \mathrm{Cl}_{2}\right)$ production in TPC plant was investigated. The lack of understanding of phenomena occurring in the reactor inspired the simulation of this work. The overall construction of the oxychlorination reactor is $3.9 \mathrm{~m}$ in diameter and $31.6 \mathrm{~m}$ high and the simulated portion is $3.9 \mathrm{~m}$ in diameter and $15.5 \mathrm{~m}$ above a distributor. The oxychlorination reactor uses ethylene, hydrochloric acid, and oxygen as the gas reactants to produce ethylene dichloride at a production rate around $28,000 \mathrm{~kg} / \mathrm{hr}$.

The CFD model is used to predict the performance of a fluidized bed on the oxychlorination reaction to produce ethylene dichloride. The CFD is a computer-based tool that can model the behavior of fluid flow. The numerical simulation in this research is based on finite volume technique in commercial code CFX 5.6. The CFD modeling of gas- 
solid flow in a fluidized bed is adapted to a Eulerian-Eulerian framework that is very suitable for density of solid.

The simulation is carried out in 2 categories; the constant catalyst volume fraction and the variation of catalyst load to study gas-solid behavior. The constant catalyst volume fraction shows pressure, temperature, and conversion profiles. The predicted values from this model no agree with the industrial data from TPC plant.

The first part studied on many effects of the boundary condition because it can be a very versatile tool to improve the operating conditions in a real plant. The effects of difference parameters on the behavior of the system were investigated. The significant discovery is the effect of feed molar distribution. The operating points where ethylene reached the highest conversion with least excess of hydrochloric acid and oxygen was at a feed of ethylene mole fraction equal to 0.22. An outlet pressure in the range of 200 to $450 \mathrm{kPa}$ boosts the ethylene conversion from 96.39 to $98.46 \%$. The improvement of ethylene conversion by increase of pressure appeared to interfere with the overall operating pressure and the gas-solid flow behavior. The increase of cooling medium temperature from 300 to $420 \mathrm{~K}$ raises the ethylene conversion from 96.9 to $98.8 \%$ and gas outlet temperature from 422 to $565 \mathrm{~K}$. Therefore, the improvement in ethylene conversion should be the adjustment of a high cooling medium temperature. Finally, the gas feed temperature has a minor effect on ethylene conversion. Although the parameter variation in the operation increased the ethylene conversion this also affects the system behavior such as the operating pressure, operating temperature, and the gas-solid flow behavior. Hence, the parameter adjustment should be considered under these limited parameter values.

The second part focused on the hydrodynamic of gas-solid flow on the reactor. The simulations were varied by catalyst quantity into 4 cases; case $1(19,500 \mathrm{~kg})$, case $2(39,000 \mathrm{~kg})$, case $3(58,500 \mathrm{~kg})$, and case $4(78,000 \mathrm{~kg})$. The profiles of temperature, catalyst volume fraction, catalyst velocity, and gas velocity at many vertical and horizontal planes were illustrated. The results showed the comparison of pressure drop, catalyst velocity, and catalyst volume fraction along the reactor height. This was useful to predict the adjustment of the catalyst quantity.

In conclusion, the CFD model based on two-fluid model was used to investigate the flow behavior of gas-solid flow and reaction in oxychlorination units. The simulation gave valuable information about dynamic behavior in fluidized bed reactors such as catalyst distribution, velocity profile of gas and catalyst, temperature and pressure contour, and average product temperature at the outlet. The results showed a very good agreement with the industrial data. The effects of many parameters were also studied. It was found that the effects of molar feed distribution, outlet pressure, and cooling medium temperature played an important role in the operation.

\section{ACKNOWLEDGEMENT}

Funding of this work has been provided jointly by the ADB Program, Chemical Engineering Department, Kasetsart University and the Graduate School, Kasetsart University. Acknowledgement is also given to Thai Plastic and Chemical (TPC).

\section{NOTATION}

$\begin{array}{ll}C_{c} & \text { Concentration of cupric chloride in catalyst, } \mathrm{mol} / \mathrm{m}^{3} \\ C_{E} & \text { Concentration of ethylene in gas phase, } \mathrm{mol} / \mathrm{m}^{3} \\ h_{\alpha \beta} & \text { Overall heat transfer coefficient, } \mathrm{kg} / \mathrm{s}^{3} \mathrm{~K} \\ h_{\alpha} & \text { Sensible enthalpy, } \mathrm{m}^{2} / \mathrm{s}^{2} \\ K_{a} & \text { Adsorption equilibrium constant, } \mathrm{m}^{3} / \mathrm{mol} \\ k_{r} & \text { Chemical reaction rate constant, } 1 / \mathrm{s} \\ r & \text { Rate of reaction, } \mathrm{kg} \mathrm{mol} / \mathrm{s} \mathrm{m}^{3} \\ M_{S} & \text { Total force on solid phase due to interaction with other phases, } \mathrm{kg} \mathrm{m} / \mathrm{s}^{2} \\ M_{\alpha} & \text { Total force on phase } \alpha \text { due to interaction with other phases, } \mathrm{kg} \mathrm{m} / \mathrm{s}^{2} \\ p & \text { Pressure, } \mathrm{kg} / \mathrm{m} \mathrm{s} \mathrm{s}^{2} \\ P_{f} & \text { Fluid phase pressure, } \mathrm{kg} / \mathrm{m} \mathrm{s}^{2} \\ P_{s} & \text { Solids pressure, } \mathrm{kg} / \mathrm{m} \mathrm{s} \mathrm{s}^{2} \\ Q_{\alpha} & \text { Interphase heat transfer to phase } \alpha \text { across interfaces with other phases, } \mathrm{kg} / \mathrm{m} \mathrm{s}^{3} \\ r & \text { Volume fraction } \\ S_{\alpha} & \text { External heat sources, } \mathrm{kg} / \mathrm{m} \mathrm{s}^{3}\end{array}$




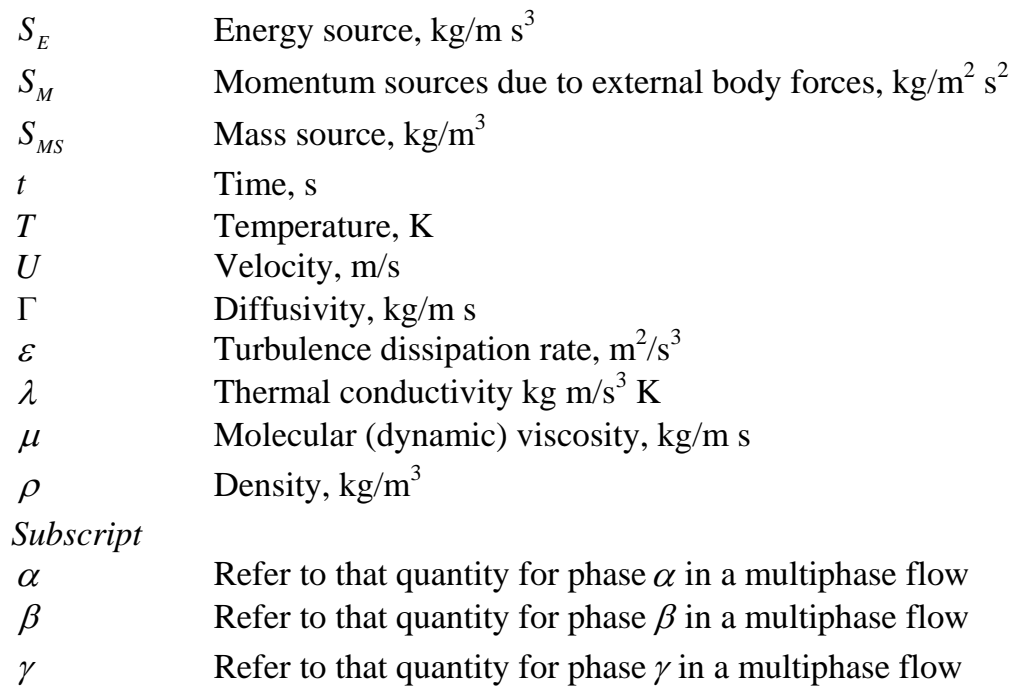

\section{REFERENCES}

[1] El-Hawagi, Mahmoud M., El-Rifai, Mahmoud A., Mathematical modeling of catalytic fluidized-bed reactors-I. The multiphase three-phase model. Chemical Engineering Science, vol. 43, no. 9, pp. 2477-2486, 1988.

[2] Van Wachem, B.G.M., Almstedt, A.E., Methods for multiphase computational fluid dynamics. Chemical Engineering Journal, vol. 96, pp. 81-98, 2003.

[3] Nawroski, J.S., Velez, E.S., Oxychlorination of ethylene. Applied Industrial Catalysis, pp. 1,239-273, 1983

[4] Wachi, W., Asai, Y., Kinetics of 1,2 Dichloroethane formation from ethylene and cupric chloride. Industrial Engineering and Chemistry Research, vol. 33, pp. 2259-264, 1994.

[5] Al-Zahrani, S.M., Aljodia, A.M., Wagialla, K.M., Modeling and simulation of 1,2-dichloroethane production by ethylene oxychlorination in fluidized-bed reactor. Chemical Engineering Science, vol. 56, pp. 621-626, 2001.

[6] Ranade, V.V., Pushing the limits of existing reactor hardware using CFD. Current Sci., vol. 77, pp. 1303-1310, 1996

[7] Gustavsson, M., Almstedt, A.E., Numerical simulation of fluid dynamics in fluidized bed with horizontal heat exchanger tubes. Chemical Engineering Science, vol. 55, pp. 857-866, 2000.

[8] Radade, W., V.V., Computational flow modeling for chemical reactor engineering. Academic Press. USA, 2002.

[9] Gidaspow, D., Multiphase flow and fluidization: Continuum kinetic theory descriptions. Academic Press. New York, 1994. 